\title{
SEFDM: Spectral Efficiency Upper Bound and Interference Distribution
}

\author{
Hedaia Ghannam and Izzat Darwazeh \\ Department of Electrical and Electronics Engineering \\ University College London, London, United Kingdom \\ E-Mail: hedaia.ghannam.15@ucl.ac.uk, i.darwazeh@ucl.ac.uk
}

\begin{abstract}
This work explores the statistical model for a 5G and beyond (5GB) multi-carrier signal candidate; spectrally efficient frequency division multiplexing (SEFDM), in which the orthogonality between its subcarriers is intentionally violated compared to conventional orthogonal frequency division multiplexing (OFDM). A mathematical model describing the statistical characteristics of inter-carrier interference (ICI) is derived and verified through system model simulations. Results show that the ICI is of normal distribution, whose variance is dependent on both the level of bandwidth compression and on the signal level. Models developed are used to derive the error bounds of SEFDM signals in Gaussian noise channels, as well as the upper bound of SEFDM spectral efficiency.
\end{abstract}

\section{INTRODUCTION}

Future cellular networks promise to deliver a gigabit experience for mobile users and machines. To meet this promise, the limited-spectrum utilisation is to be improved [1]. One contribution in this direction was the development of spectrally efficient frequency division multiplexing (SEFDM), which was first proposed in 2003 [2].

SEFDM is a multi-carrier system that achieves a spectral efficiency gain through packing the subcarriers closer together by compromising the orthogonality of orthogonal frequency division multiplexing (OFDM). The multi-stream faster than Nyquist (FTN) technique proposed in [3], is SEFDM's time domain counterpart and has similar spectral efficiency gains. A survey of SEFDM work and research is reported in CSNDSP 2018 conference [4].

Significant spectral efficiency gains with error performance of SEFDM being reasonably close to OFDM, were demonstrated in experimental SEFDM test beds for wireless [5], optical/mm-wave [6], VLC [7], very high speed optical systems [8] [9] and the downlink for Internet of Things (IoT) networks [10].

Spectral efficiency upper-bound and capacity are key performance metrics to evaluate the benefits of SEFDM and find the scenarios where SEFDM will be beneficial in comparison to OFDM. The capacity of SEFDM with different pulse shapings has been demonstrated in [11] from a theoretical view point, without considering the practicality of the systems used. This work extends the work reported in [9], where the capacity of SEFDM has been derived, showing that SEFDM capacity depends on the interference level between its subcarriers.

To the best of our knowledge, this paper for the first time provides a thorough insight into the statistical nature of inter- carrier interference (ICI) for SEFDM, which is required to calculate the spectral efficiency upper-bound.

\section{SEFDM WAVEFORM}

SEFDM is a multi-carrier modulation technique, where symbols are generated similarly to OFDM by means of modified inverese fast Fourier transmform (IFFT) structures [6]. Let $\mathbf{z} \in \mathbb{C}^{N \times 1}$, be the complex baseband symbols to be modulated by $N$ subcarriers of SEFDM signal, then the discrete SEFDMmodulated signal can be expressed as [12]

$$
\mathbf{x}=\frac{1}{\sqrt{Q}} \sum_{n=0}^{N-1} z_{n} \exp \left(j 2 \pi \alpha \frac{q n}{Q}\right), 0 \leq q \leq Q-1
$$

where $\alpha \in(0,1]$ is the compression factor, $Q \geq N$ is the total number of samples and $1 / \sqrt{Q}$ is employed for normalization purposes.

If the number of subcarriers for the case of OFDM $(\alpha=1)$ is given by $N_{o}$, then, due to spacing compression in SEFDM, more subcarriers will occupy the same bandwidth. The relation

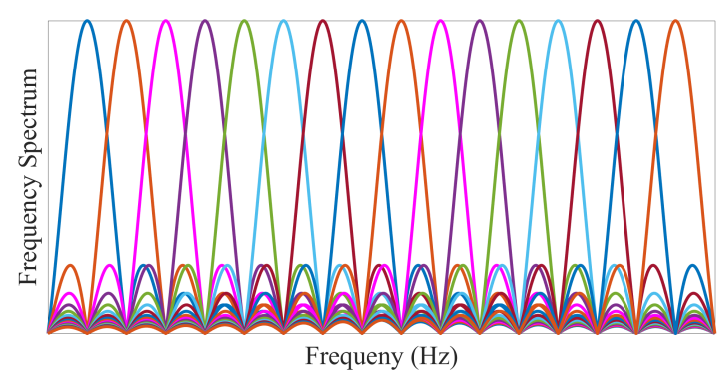

(a)

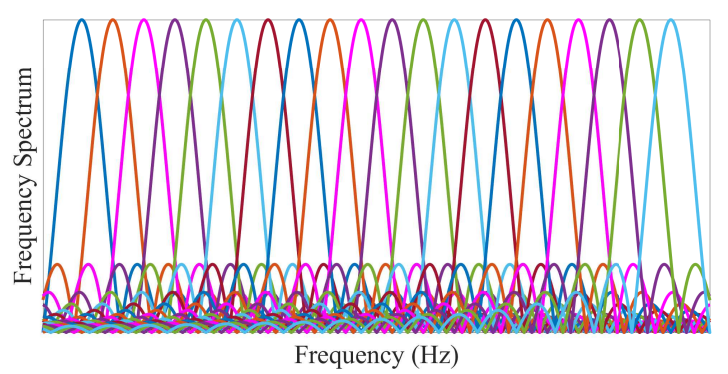

(b)

Fig. 1: The frequency spectrum of (a) OFDM $(\alpha=1), N_{o}=$ 16 (b) SEFDM ( $\alpha=0.8), N=20$. 
is given by $N=\left\lfloor N_{o} / \alpha\right\rfloor$, where $\lfloor$.$\rfloor denotes the floor$ operation. Fig. 1. shows a frequency spectrum of rectangular pulses with $\alpha=0.8, N_{o}=16$ and $N=20$. Resultantly, the data rate increases by a factor of $(1-\alpha) / \alpha \times 100 \%$, which is $25 \%$ for this case.

If the SEFDM signal in (1) were to be transmitted over an additive white Gaussian noise (AWGN) channel (w), with zero mean and variance $\sigma_{n}^{2}=N_{0} / 2$, where $N_{0}$ is the noise power spectral density, the SEFDM-received signal $\mathbf{y}$ can be presented by

$$
\mathbf{y}=\mathbf{x}+\mathbf{w}
$$

Fig. 2 shows the SEFDM system transceiver block diagram. At the receiver front end, an FFT is used as a matched filter to demodulate the signal affected by the channel and contaminated by interference, resulting from the non-orthogonal nature of the SEFDM signal. The estimated SEFDM symbol ( $\hat{\mathbf{z}})$ can be expressed as [12]

$$
\hat{\mathbf{z}}=\mathbf{\Lambda} \mathbf{z}+\mathbf{w},
$$

where $\Lambda$ is the correlation matrix of size $N \times N$, with a diagonal of ones and non-diagonal elements $\left(\Lambda_{m, n}\right)$ representing the correlation between two subcarries $m$ and $n$ given by [12]

$$
\begin{aligned}
\Lambda(m, n)= & \exp (j \pi \alpha(m-n)) \times \\
& \exp \left(\frac{-j \pi \alpha(m-n)}{Q}\right)\left[\frac{\operatorname{sinc}(\alpha(m-n))}{\operatorname{sinc}\left(\frac{\alpha(m-n)}{Q}\right)}\right] .
\end{aligned}
$$

For a detailed mathematical treatment readers are referred to [12]. The next section studies the statistical nature of the SEFDM ICI given in (4).

\section{The Statistical ChaRACTERISTICS OF ICI IN SEFDM}

As the main focus of this work is the ICI within the SEFDM symbol, two assumptions are made: i) zero intersymbol interference (ISI) between adjacent SEFDM symbols due to the existence of long guard-band or cyclic prefix; ii) the channel is noiseless (i.e. $\mathbf{w}=0$ in (3)).
It is noteworthy that the ICI term $(\Lambda)$ on its own, given in (4), is deterministic for a given set of system parameters. In (3), this term is multiplied by the transmitted symbol $z$, which belongs to a random variable set $Z$, thus, the resultant $\hat{z}$ will belong to a random variable set too. According to the central limit theorem, if the number of interfering signals is high (i.e. relatively high $N$ ), then, the interference can be approximated to a Gaussian process. Consequently, ICI can be modelled by a Gaussian process characterised by its first and second moments, which are the mean and variance, respectively.

In the following, the symbol $z_{n}$ modulating the $n^{\text {th }}$ subcarrier belongs to a QPSK random variable set $Z=\{1+j, 1-$ $j,-1+j,-1-j\}$ of mean $\mu_{z}=0$. Multiplying the random variable $Z$ by a constant value $\Lambda$ in (3), results in multiplying the mean of $Z$ by that constant. Hence, the ICI mean will be $\mu_{I}=0$.

The ICI variance $\sigma_{I, n}^{2}$ caused by interference on the $n^{\text {th }}$ subcarrier from all other subcarriers is [13]

$$
\sigma_{I, n}^{2}=\sum_{m=0, m \neq n}^{N-1} \sigma_{m, z}^{2} \times\left|\Lambda_{m, n}\right|^{2},
$$

where $\sigma_{m, z}^{2}$ is the variance of the data symbols $z$. Consequently, the total ICI variance within one SEFDM symbol is equal to

$$
\sigma_{I}^{2}=\sum_{n=0}^{N-1} \sigma_{I, n}^{2}
$$

For normalization purposes, if the total variance is divided by the transmitted signal power $\left(N \times \sigma_{z}^{2}\right)$, then the scalar quantity $\tilde{\sigma_{I}}$ presents the normalized variance function. Therefore, if the signal is transmitted with power $P$, then, the total interference in this case will be $\left(P \times \tilde{\sigma_{I}}\right)$ Watt.

The accuracy of the proposed ICI statistical model can be examined by simulating the system of Fig. 2 and then plotting the probability density function (PDF) of the received symbols $\hat{\mathbf{z}}$ in (3) without noise (i.e. $\mathbf{w}=0$ ) and $P=1$ Watt $\left(\sigma_{I}^{2}=\tilde{\sigma_{I}^{2}}\right)$. Fig. 3(a) shows the histogram of $\Re\{\hat{\mathbf{z}}\}$ for the case of QPSK symbols transmission, where $N=128$ and $\alpha=0.8$. Expectedly, this has a normal distribution and using

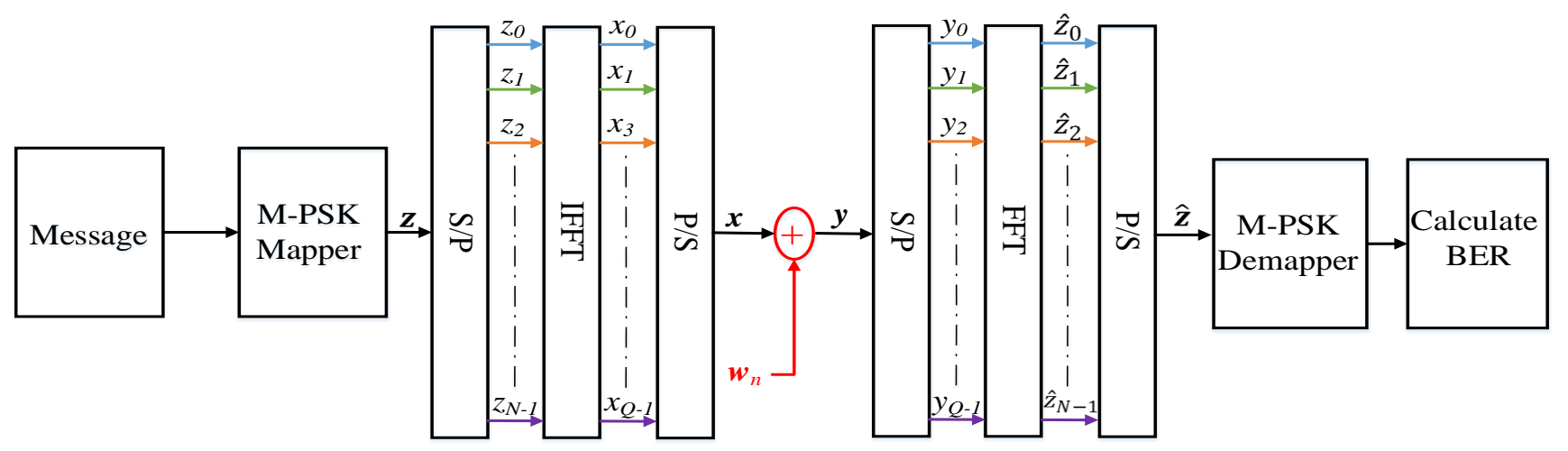

Fig. 2: Transceiver block diagram. 


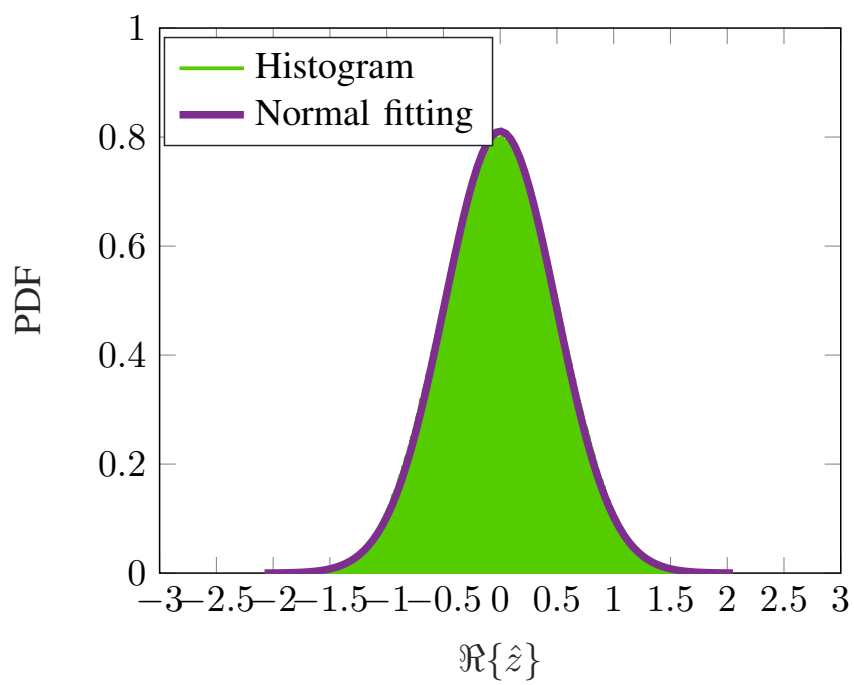

(a) PDF of ICI, $\alpha=0.8, N=128$, zero noise.

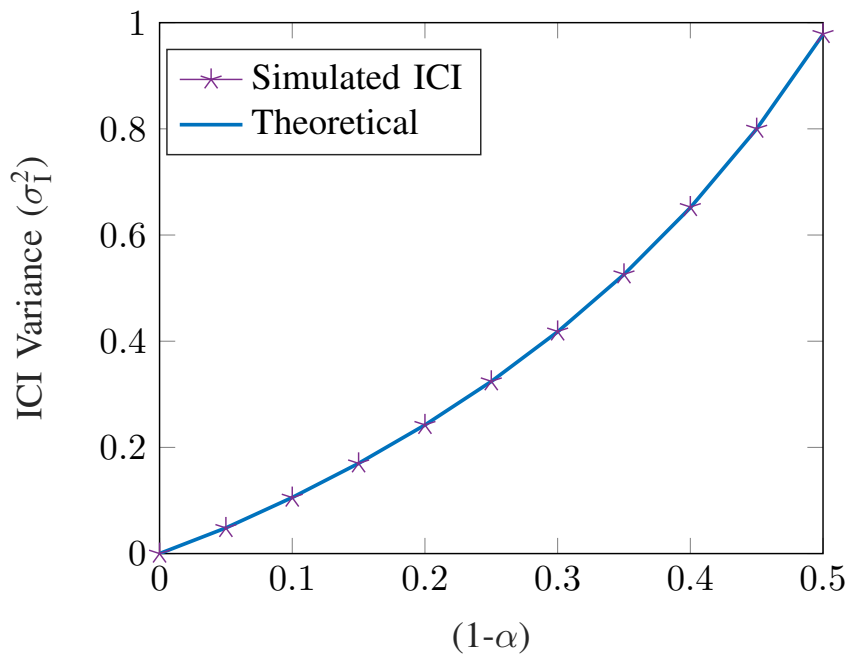

(b) $\sigma_{I}^{2}, N=128$.

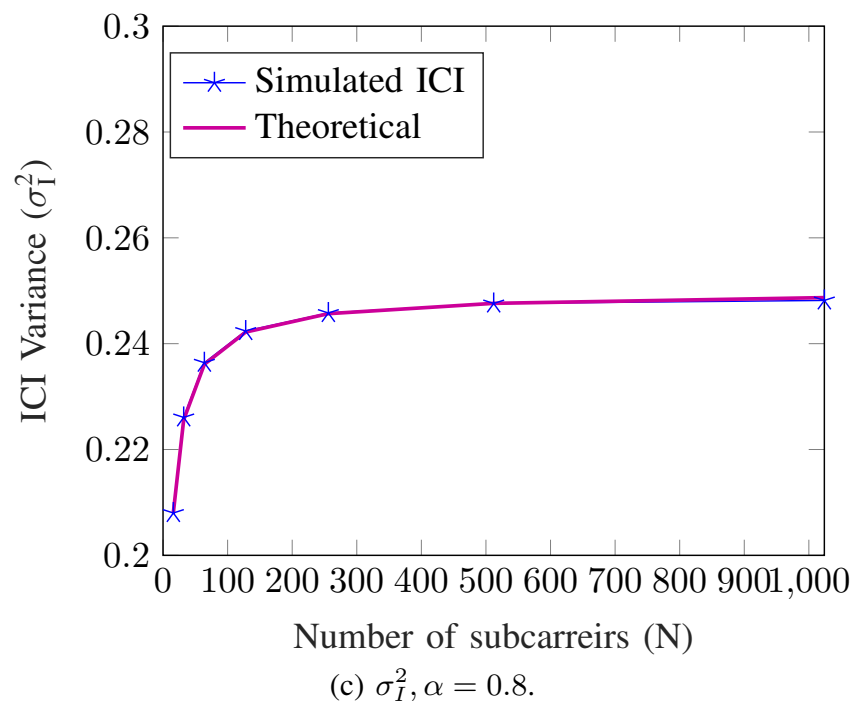

Fig. 3: ICI statistical model examination. the MATLAB's distribution fitting tool, the mean and variance are 0 and 0.2422 , respectively. Furthermore, the effects of $\alpha$ and $N$ on the variance $\sigma_{I}^{2}$ are tested individually, by fixing one of them at a time as shown in Fig. 3(b) and (c). The simulated and theoretical results using the derived equations (5) and (6) match. Clearly, $N$ doesn't play a major role on the value of $\sigma_{I}^{2}$ (i.e. $10 \%$ in the worst case), unlike the effect of $\alpha$ (above $90 \%$ ), because the ICI mainly comes from the neighbouring subcarriers on both sides of a given subcarrier.

To sum up the results of $\sigma_{I}^{2}$, Fig. 4 shows the variance for $\alpha \in[0.5,1)$ and $N \in\{16,32,64,128,256,512,1024\}$. Fig. 4 will be used in the next sections to calculate the error bounds and the upper bounds for spectral efficiency of SEFDM signals.

\section{PRoBABILITY OF ERROR BoUndaRIES}

The probability distribution function (PDF) of the combined AWGN and ICI impairments will also be Gaussian. The mean and variance of this Gaussian PDF is the summation of AWGN and ICI means and variances [13]. For instance, refer to Fig. 3 (a) and given the white noise mean is zero, while the variance is set to be constant $\left(\sigma_{n}^{2}=0.2\right)$, the total variance of Fig. 5 is $\sigma_{t}^{2}=0.4423$ and this is approximately the summation of $\sigma_{n}^{2}=0.2, \sigma_{I}^{2}=0.2422$.

When a matched filter is used at the receiver front end, which is presented by an FFT in Fig. 2, the theoretical probability of error $\left(P_{e}\right)$ value for QPSK-OFDM is given by [14]

$$
P_{e}=Q\left(\sqrt{\frac{2 E_{b}}{N_{0}}}\right),
$$

where $E_{b} / N_{0}$ is the ratio of energy per bit over the noise spectral density and $Q($.$) is the tail distribution function [14].$

However, for $\alpha<1$, the probability of error is higher, because the total variance of the received signal increases due to ICI as shown before. Therefore, the $P_{e}$ for this specific case becomes

$$
P_{e}=Q\left(\sqrt{\frac{2 E_{b}}{N_{0}+2 E_{b} \times \tilde{\sigma}_{I}^{2}}}\right) .
$$

Clearly (8) turns into (7) when $\alpha=1$ as $\tilde{\sigma_{I}^{2}}=0$. To prove the validity of (8), $P_{e}$ is simulated for the system in Fig. 2 and compared to the theoretical results for Q-PSK symbols transmission and $N=128$ in Fig. 6. The following can be observed from the results: $i$ ) the theoretical prediction (diamonds marks) matches the simulation results (stars marks); ii) an error floor appears in SEFDM. This is because, as shown in (8), the interference level increases by increasing the signal power. Thus, unlike white noise, the ICI variance is signal dependent; iii) the error floor increases by increasing the compression level of SEFDM signals. To mitigate the ICI effect, a more sophisticated and complex receiver is required; such as a sphere decoder [8] or a turbo equaliser for the case of coded systems [15] [16]. The ideal scenario is to remove the interference completely in (8), then, the error rate of SEFDM will be identical to OFDM. The next section calculates the 


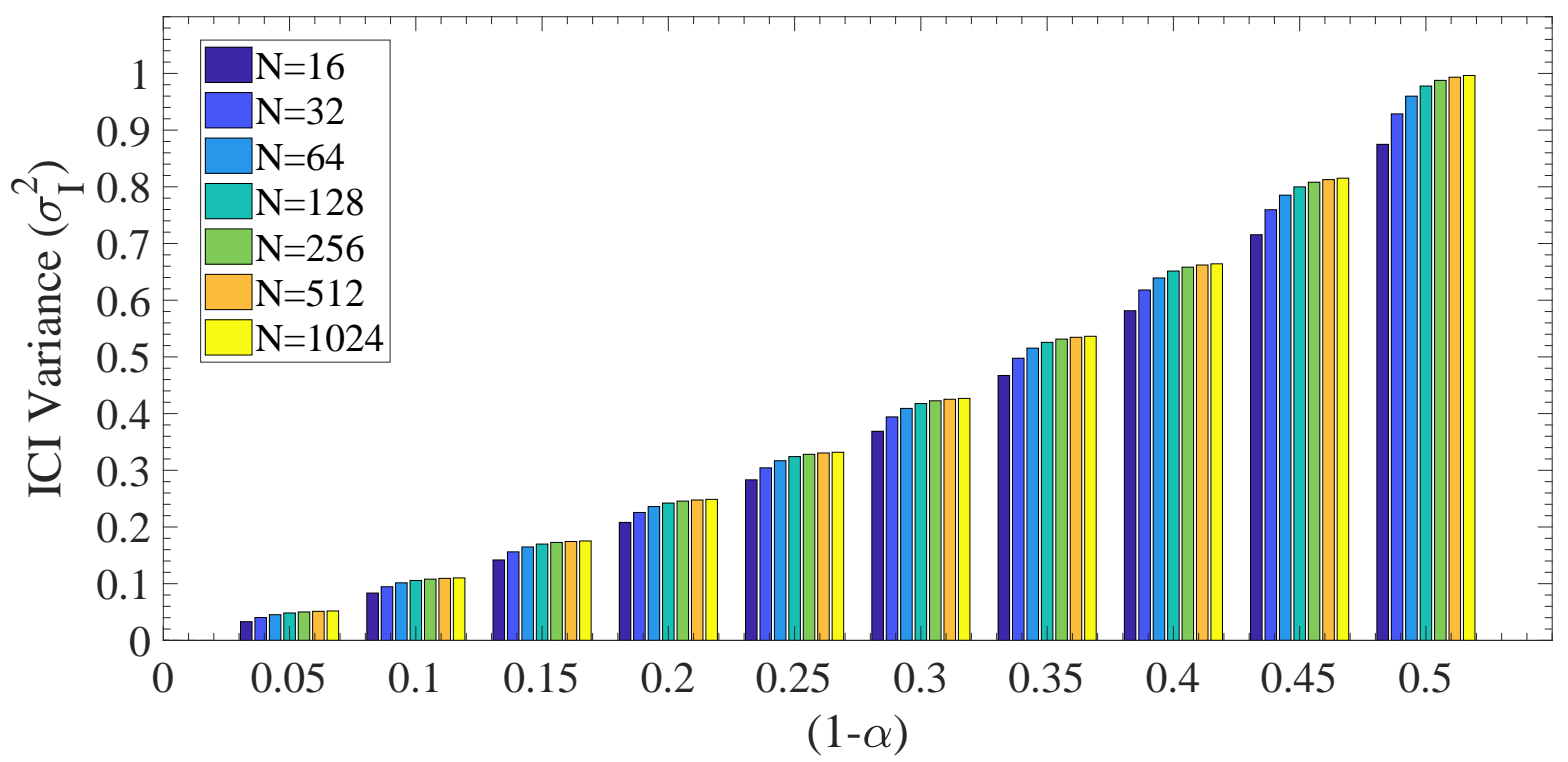

Fig. 4: The ICI variance for different $\alpha$ and $N$.

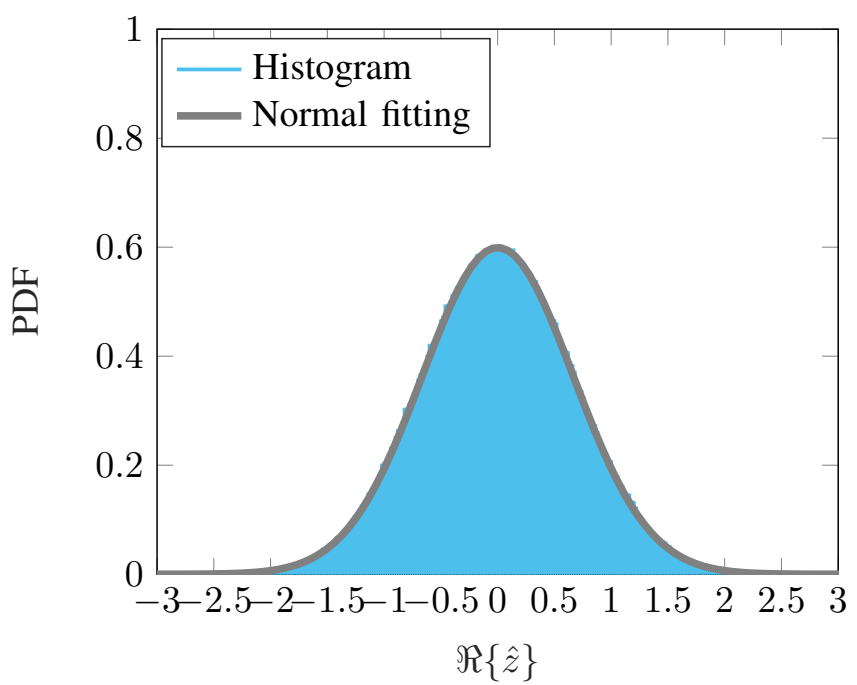

Fig. 5: PDF of the received signal with $\alpha=0.8, N=128$ and $\sigma_{n}^{2}=0.2$.

spectral efficiency upper bound for SEFDM with and without ICI cancellation.

\section{SEFDM SPECTRAL EFFICIENCY UPPER BOUND}

Conceptually, the capacity of a communication system has to be calculated at the front end of its detector, which for the case of SEFDM is at the input of the M-PSK demapper. For instance, consider an OFDM signal with a transmission rate equal to the Nyquist rate (i.e. $R_{s}=2 B$ sample/sec), where $R_{s}$ is the sample rate and $B$ is the channel bandwidth. The

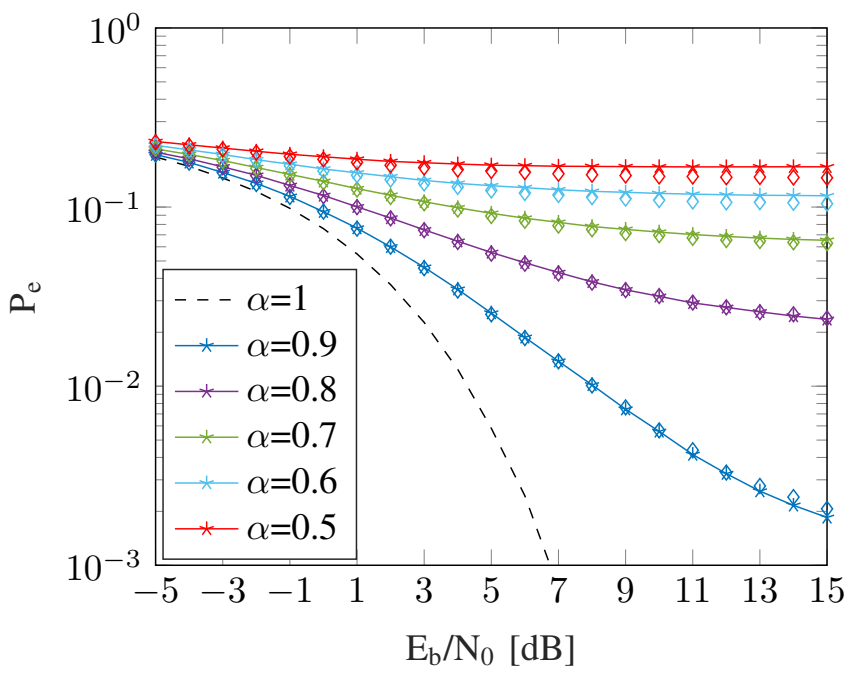

Fig. 6: $P_{e}$ vs. $E_{b} / N_{0}$ for QPSK-SEFDM signals, $N=128$.

channel capacity $(C)$, based on Shannon's original paper [17], in bits per use is

$$
C=\frac{1}{2} \log _{2}\left[1+\frac{P}{\left(N_{0} / 2\right) \times B}\right] \text { bits per use, }
$$

where $P$ is the transmitted power level. As mentioned before in section II, SEFDM uses more subcarriers compared to OFDM within the same channel bandwidth due to the compression of the subcarriers (i.e. $N>N_{o}$ ). However, for a fair comparison, the total transmitted power for OFDM and SEFDM must be equal. Therefore, the power per subcarrier for SEFDM is reduced by a factor $N / N_{o}[11]$. 


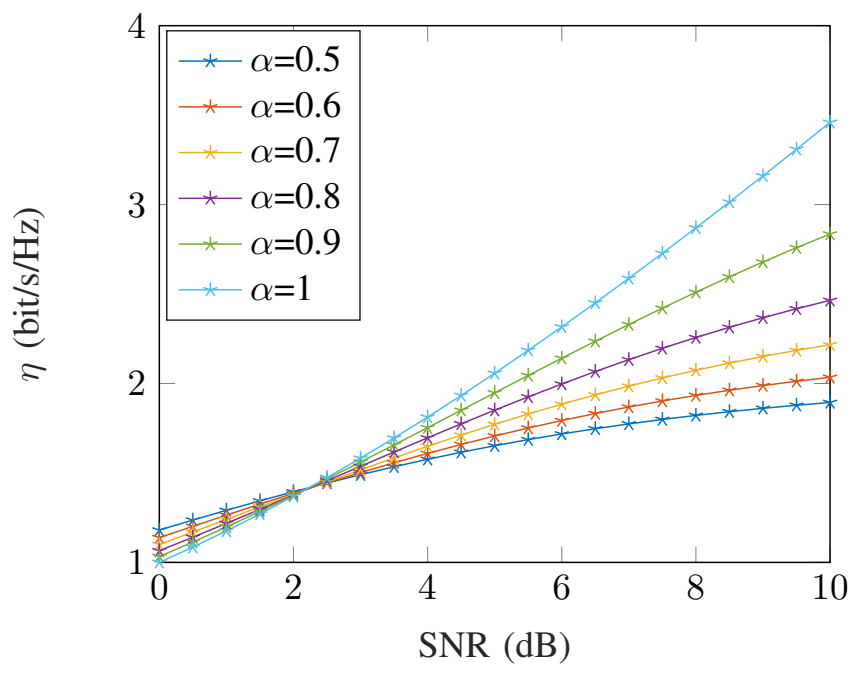

(a)

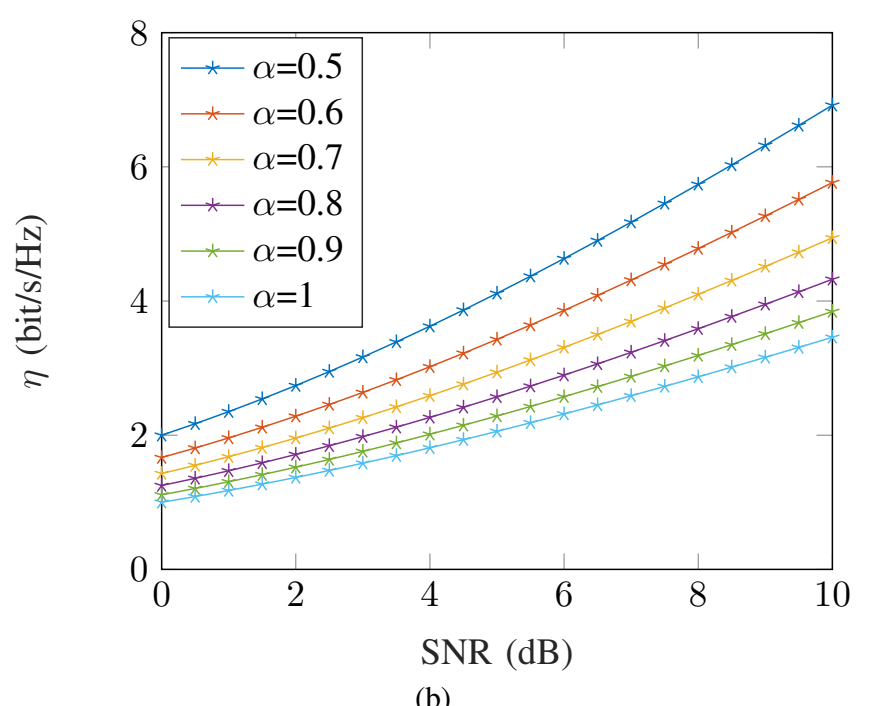

(b)

Fig. 7: $\eta$ vs. SNR for QPSK-SEFDM signals, $N=128$, without/with ICI cancellation.

The multiplication of (9) by the number of times the channel is used per unit time gives the capacity in bit/s [17]. This factor is equal to the ratio of: number of samples transmitted within time $T$ to this time duration $T$. Owing to the increase in the number of subcarriers in SEFDM, the number of samples transmitted in $T$ seconds is equal to $\left(R_{s} \times T / \alpha\right)$ samples, thus, the system capacity is

$$
C=\frac{B}{\alpha} \log _{2}\left[1+\frac{P}{\left(N_{0} / 2\right) \times B+P \times \tilde{\sigma}_{I}^{2}}\right] \mathrm{bit} / \mathrm{s},
$$

where the interference of SEFDM appears here as $P \times \sigma_{I}^{2}$. The spectral efficiency $(\eta)$ in $(\mathrm{bit} / \mathrm{s} / \mathrm{Hz})$ is found by dividing the capacity of (10) by the channel bandwidth

$$
\eta=\frac{1}{\alpha} \log _{2}\left[1+\frac{P}{\left(N_{0} / 2\right) \times B+P \times \tilde{\sigma_{I}^{2}}}\right] \mathrm{bit} / \mathrm{s} / \mathrm{Hz} .
$$

In Fig. 7(a), $\eta$ is plotted versus signal to noise ratio $(\mathrm{SNR}=$ $P /\left(\left(N_{0} / 2\right) B\right)$ without ICI cancellation. The results can be divided into two regions: $i$ ) low SNR region, where the noise is dominant and SEFDM spectral efficiency is higher than that of OFDM; ii) high SNR region, where the ICI is dominant and it limits the spectral efficiency of SEFDM, with no advantage of transmitting with higher SNR.

This information is important when considering the utility of SEFDM. For instance, powerful channel coding; such as turbo coding and LDPC, allow reliable information transmission with low SNR. Thus, SEFDM in such a case is more efficient when compared to OFDM.

Conversely, by considering the ideal case of $100 \%$ ICI elimination in (11), the achievable $\eta$ becomes as shown in Fig. 7(b). Clearly, the capacity advantage of SEFDM is higher with ICI cancellation, especially in high SNR regions.

\section{CONCLUSIONS}

In this work and for the first time, a thorough study of the statistical distribution of ICI in SEFDM is presented. Thereafter, the statistical model is used to calculate the error lower bound and capacity for SEFDM signals. The results indicate that the potential upper-bound of SEFDM spectral efficiency is higher than that of OFDM, at the expense of more complex receivers.

\section{ACKNOWLEDGMENT}

This work has been supported in part by the EU Funded 5GExchange Innovation Project 5GEx (www.5gex.eu). We are grateful to UCL as well for funding Hedaia Ghannam's PhD studies through ORS and the UCL Dean of Engineering Sciences Award.

\section{REFERENCES}

[1] F. Luo and C. Zhang, Signal Processing for 5G: Algorithms and Implementations, ser. Wiley - IEEE, 2016.

[2] M. Rodrigues and I. Darwazeh, "A Spectrally Efficient Frequency Division Multiplexing Based Communications System," in Proc. 8th Int. OFDM Workshop, Hamburg, 2003, pp. 48-49.

[3] F. Rusek and J. Anderson, "Multistream Faster than Nyquist Signaling," IEEE Trans. Commun., vol. 57, no. 5, pp. 1329-1340, May 2009.

[4] I. Darwazeh, H. Ghannam, and T. Xu, "The First 15 Years of SEFDM: A Brief Survey," in CSNDSP 2018 Conf. (accepted), June 2018.

[5] T. Xu and I. Darwazeh, "Transmission Experiment of Bandwidth Compressed Carrier Aggregation in a Realistic Fading Channel," IEEE Trans. Veh. Technol., 2016.

[6] T. Xu, S. Mikroulis, J. E. Mitchell, and I. Darwazeh, "Bandwidth Compressed Waveform for $60-\mathrm{GHz}$ Millimeter-Wave Radio over Fiber Experiment," J. of Lightwave Technol., vol. 34, no. 14, pp. 3458-3465, July 2016.

[7] Y. Wang, Y. Zhou, T. Gui, K. Zhong, X. Zhou, L. Wang, A. P. T. Lau, C. Lu, and N. Chi, "SEFDM Based Spectrum Compressed VLC System Using RLS Time-domain Channel estimation and ID-FSD Hybrid Decoder," in 42nd ECOC 2016, Sept 2016, pp. 1-3.

[8] D. Nopchinda, T. Xu, R. Maher, B. C. Thomsen, and I. Darwazeh, "Dual Polarization Coherent Optical Spectrally Efficient Frequency Division Multiplexing," IEEE Photon. Technol. Lett, vol. 28, no. 1, pp. 83-86, Jan 2016 . 
[9] J. Zhou, Y. Qiao, Z. Yang, M. Guo, and X. Tang, "Capacity Limit for Faster-than-Nyquist Non-Orthogonal Frequency-Division Multiplexing Signaling," in Scientific Reports, 2017.

[10] T. Xu and I. Darwazeh, "Non-Orthogonal Narrowband Internet of Things: A Design for Saving Bandwidth and Doubling the Number of Connected Devices," IEEE Internet Things J., pp. 1-1, 2018.

[11] D. Rainnie, Y. Feng, and J. Bajcsy, "On Capacity Merits of Spectrally Efficient FDM," in MILCOM 2015, Oct 2015, pp. 581-586.

[12] S. Isam and I. Darwazeh, "Characterizing the Intercarrier Interference of Non-orthogonal Spectrally Efficient FDM System," in 2012 8th CSNDSP, Poznan, July July 2012, pp. 1-5.

[13] L. L. Hanzo, C. H. Wong, and M. S. Yee, Adaptive Multicarrier Modulation. Wiley-IEEE Press, 2002.

[14] J. G. Proakis, Digital communications, ser. McGraw-Hill series in electrical and computer engineering. New York: McGraw-Hill, 1995.

[15] H. Ghannam and I. Darwazeh, "Signal Coding and Interference Cancellation of Spectrally Efficient FDM Systems for 5G Cellular Networks," in 2017 24th ICT Conf., May 2017, pp. 1-6.

[16] _ - "SEFDM over satellite systems with advanced interference cancellation," IET Communications, vol. 12, no. 1, pp. 59-66, 2018.

[17] C. E. Shannon, "Communication in the Presence of Noise," Proceedings of the IRE, vol. 37, no. 1, pp. 10-21, Jan 1949. 